\title{
Isotropic shear bond strength behavior of superficial bovine dentin: A pilot study
}

\author{
Camila Sabatini*, Sebastiano Andreana \\ Department of Restorative Dentistry, School of Dental Medicine, State University of New York, Buffalo, USA \\ Email: *cs252@buffalo.edu
}

Received 20 January 2013; revised 22 February 2013; accepted 28 February 2013

\begin{abstract}
The aim of this pilot study was to evaluate the shear bond strength of superficial bovine incisor dentin in different crown regions. Bonding was performed to the incisal, middle and cervical thirds of superficial bovine coronal dentin $(n=20)$ with a two-step etchand-rinse adhesive (Optibond Solo Plus) and resin composite (Z100). Shear bond strength was evaluated at $24 \mathrm{~h}$ and failure modes of representative specimens wereobserved with FE-SEM. Non-parametric Kruskal-Wallis test with a significance level of 0.05 was used for data analysis. Mean shear bond strength values for the incisal, middle, and cervical thirds were 36.9 (3.1), 42.6 (2.6), and 37.1 (2.1) respectively with no significant differences evidenced between the crown thirds $(p=0.19)$. Observation of the failure mode of representative specimens demonstrated that specimens with high bond strength values exhibited-predominantly mixed-type failures whereas low strength specimens exhibited adhesive failures between the dentin and adhesive. The absence of significant differences in shear bond strength between crown thirds indicate that, regardless of tubule orientation, any crown region can be used when superficial bovine incisor dentin is used for shear bond strength testing.
\end{abstract}

Keywords: Adhesion; Shear Bond Strength; Bovine Dentin; Isotropic Properties; Failure Mode

\section{INTRODUCTION}

Adhesion to tooth structure, in particular to dentin, has been an area of extensive research in the last few decades [1-3]. The mechanism of hybridization by which resin monomers infiltrate the partially demineralized collagen network is highly complex and dependent on a number of variables [4], of which the substrate, particularly dentin, is perhaps the single most critical aspect due to the

${ }^{*}$ Corresponding author. large morphological variability known to exist between different teeth and in various regions of the same tooth [5]. The standards for bond strength testing developed by the International Organization for Standardization (ISO) provide a set of guidelines to facilitate reproducibility of the testing conditions and thus, allow comparisons among studies (ISO/TR 11405). However, despite standardization efforts, variability in the testing conditions still exists particularly in aspects relative to the substrate and testing conditions [6].

Bond strength studies commonly use human third molars or bovine incisors as bonding substrate. Despite some anatomical and permeability differences [7], histochemical and morphological studies have shown that human and bovine dentin are essentially the same [8-11]. Studies have reported no differences in the concentration of tubules per $\mathrm{mm}^{2}$ between human and bovine coronal dentin [11,12]. However, compared to human molars, dentinal tubules from bovine incisors have been reported to be of larger size and have more porous intertubular dentin [13]. In this regard, according to Nakamichi, only superficial bovine dentin may be considered a suitable substitute to human dentin [9].

Dentin is a complex mineralized hydrated tissue that makes up the bulk of the tooth. It is composed primarily of type I collagen and nano-crystalline apatite mineral [5]. Orientation of the collagen fibers, dentin's main structural component, determines largely its mechanical properties [14]. The rather simple microstructure of dentin consists of dentinal tubules surrounded by a cuff of highly mineralized peritubular dentin and less mineralized inter-tubular dentin between the tubules [15]. Dentinal tubules are arranged in a radial pattern and follow an "S" or zigzag course from the pulp chamber to the dentino-enamel junction (DEJ). This structural arrangement leads to variation in the size and number of tubules per square millimeter, as well as in the amount of inter-tubular dentin at different dentin depths [5]. It is well accepted that mechanical properties vary with distance from the pulp [16]. A number of studies have demonstrated the anisotropic mechanical properties of dentinin- 
cluding differences in bonding efficiency depending on tubule orientation and location $[4,14,17,18]$. However, most of these studies report primarily in the differences in strength at different dentin depths [19]. While differences in bond strength are known to exist at different dentin depths, it has been assumed that strength values obtained from different crown regions at one same dentin depth may be no different. The use of bovine teeth as a substrate for bond strength studies has been suggested due to their overall greater availability and considerably larger size that allows greater number of specimens to be fabricated from a single crown. However, the assumption that similar bond strengths may be obtained from different crown regions provided that the same depth in dentin is used has not been tested, and may subtract from the aforementioned advantages associated with their use. The zigzag course of the tubules from the pulp chamber to the dentin-enamel junction (DEJ), the branching as well as the interruptions in the main course of the tubular structure (author's unpublished observations) may also result in variations in tubular concentration and crosssectional view in the different crown regions even at the same dentin depth. Consequently, differences in the extent of hybridization and thus on the bond strength may exist. The authors are not aware of studies evaluating bonding efficiency in different regions of a crown at one same dentin depth.

Therefore, the objective of this pilot study was to evaluate the shear bond strength (SBS) of superficial bovine coronal dentin obtained from the incisal, middle and cervical thirds. Furthermore, observations of the failure mode of representative specimens for the different crown thirds with scanning electron microscope (FE-SEM) were also described. The null hypothesis was that there would be no significant difference in bond strength values obtained from the different crown thirds.

\section{MATERIALS AND METHODS}

\subsection{Specimen Preparation}

Ten non-carious, unrestored bovine mandibular central incisors were used to obtain dentin substrates for bonding. The incisors were used within 3 months of extraction and stored in an aqueous disinfectant $(0.5 \%$ chloramine $\mathrm{T}$ solution at $4^{\circ} \mathrm{C}$ ) until ready to be used. A slowspeed saw (Isomet, Buehler, Lake Bluff, IL, USA) with a diamond disk under cooling water was used to separate the crowns from the roots at the CEJ level. From each crown, two transverse sections were made between the incisal and middle third and between the middle and cervical third to obtain incisal, middle and cervical sections. Each fragment was further sectioned longitudinally to obtain mesial and distal halves (Figure 1). This yielded a total of 60 specimens with a sample size of twenty $(\mathrm{n}=$

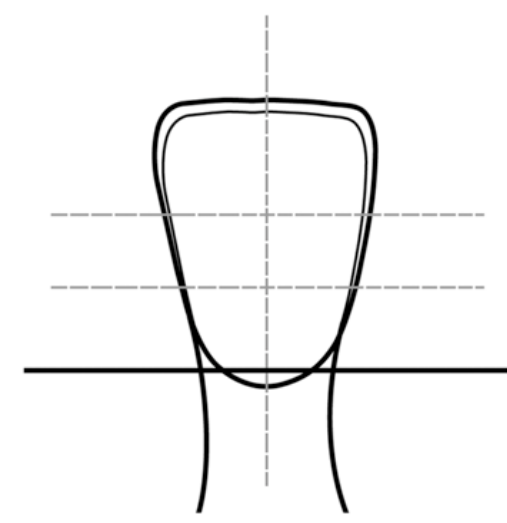

Figure 1. Diagram of the sections obtained from bovine incisors that were subsequently embedded on a chemically polymerized methacrylate.

20) per crown third. The sectioned specimens were embedded in a chemically-polymerized methacrylate (Fastray, HJ Bosworth, Skokie, IL, USA) with the facial surface exposed. The exposed surface was ground flat on a model trimmer until superficial dentin was revealed. The exposed dentin was finished with 400- and 600-grit silicon carbide (SiC) abrasive paper (Buehler). The prepared specimens were stored in de-ionized water at $4^{\circ} \mathrm{C}$ until ready to be bonded.

\subsection{Shear Bond Strength Testing}

One hour prior to bonding, the specimens were acclimatized to room temperature $\left(23^{\circ} \mathrm{C} \pm 2^{\circ} \mathrm{C}\right)$. Immediately before the start of the procedures, the specimens' surfaces were slightly refinished with the 600 -grit SiC abrasive paper to expose fresh dentin. Bonding to the different crown thirds was randomized to avoid the confounding effects of sequence of bonding. Dentin specimens were etched with 35\% phosphoric acid (Ultra-etch, Ultradent, South Jordan, UT, USA) for 15 seconds, rinsed and blot dried for moist bonding. An etch-and-rinse adhesive system Optibond Solo Plus (Kerr, Orange, CA, USA) was applied and polymerized for 20 seconds following manufacturer's instructions with a light curing unit Bluephase C8 (Ivoclar-Vivadent, Amherst, NY, USA). A minimum power density of $800 \mathrm{~mW} / \mathrm{cm}^{2}$ was ensured by periodically monitoring the unit's output with a radiometer (Demetron, Kerr). The adhesive system was selected based on our own unpublished studies indicating consistency and reliability of the product. The specimens were placed on a specially fabricated bonding jig (Ultradent) with a cylindrical mold of $2.38 \mathrm{~mm}$ in diameter. The mold was filled with resin composite Z100 (3M ESPE, Saint Paul, MN, USA) in a single increment no greater than $2 \mathrm{~mm}$ and polymerized for 40 seconds. Immediately after bonding, the specimens were stored in an incubator at $37^{\circ} \mathrm{C}$ and $100 \%$ humidity for 24 hours. 
Shear bond strength was measured using a testing machine (Ultratester, Ultradent) at a test speed of $1 \mathrm{~mm} / \mathrm{min}$ with a load cell of 1000 lbs $(453.6 \mathrm{Kg})$. A notched crosshead designed to match the diameter of the bonded specimen was used to apply the testing load. Specimens were stabilized in a testing jig, which was free to move to facilitate positioning under the load. The test base was then positioned so that the notched crosshead was placed against the specimen surface and the notch was fitted to the bonded specimen. The load required to debond the specimen was recorded and expressed in megapascals (MPa) by dividing the load by the surface area of the bonded specimen. Mean shear bond strength values were calculated. All descriptive statistics and data analysis were performed using statistical software (Stata/SE 10, StataCorp LP, Texas, USA). Because the data was not normally distributed, a non-parametric test was selected to identify significant differences in bond strength values between the different crown thirds at 0.05 level. Kruskal-Wallis rank test was used to evaluate whether the location of bonding had a significant effect on the SBS values.

\subsection{Observations on Failure Mode and Tubular Orientation}

Observations of the failure mode of representative specimens were done by a trained examiner (CS) with field emission scanning electron microscope (FE-SEM, Hitachi SU-70, Hitachi, Krefeld, Germany) in backscattered electron mode. Three representative samples corresponding to the highest, a value close to the mean and the lowest bond strength values for each crown third were observed at a magnification of $50 \times$. The fractured surfaces were classified into one of five failure modes: 1 ) cohesive in dentin; 2) adhesive at the interface between dentin and adhesive resin; 3) cohesive in adhesive resin; 4) adhesive at the interface between the adhesive resin and composite; 5) cohesive in composite; 6) mixed failure across the different layers of the interface.

Additional untreated dentin surfaces were also observed in FE-SEM to confirm dentinal tubule orientation in the different crown regions. Three bovine incisors were sectioned as previously described (Figure 1) to obtain a total of eighteen specimens with six samples corresponding to each incisal, middle and cervical thirds. The specimens were desiccated, frozen in liquid nitrogen and cryofractured with a razor blade after making a series of superficial notches at the DEJ level. The specimens were coated with a $20 \mathrm{~nm}$ layer of evaporated carbon to provide conductivity and observed under SEM.

\section{RESULTS}

The sample size for this pilot study was 60 with 20 specimens for each incisal, middle, and cervical thirds. Overall, when data from all groups was combined, the mean SBS was 38.8 (1.5). The mean shear bond strength and standard error values for the incisal, middle, and cervical thirds were 36.9 (3.1), 42.6 (2.6), and 37.1 (2.1) respectively (Table 1). When the individual values were considered, the highest bond strength value was obtained for the cervical third and the lowest SBS value for the middle third with values of 59.8 $\mathrm{MPa}$ and 17.3 MPa respectively. The non-parametric Kruskal-Wallis rank test did not detect statistically significant differences in mean SBS values among the different coronal dentin locations (chi-square $=3.32, \mathrm{p}=0.19$ ) (Table 2).

Observations of the failure mode of representative specimens for the different crown thirds are summarized in Table 3. Overall, the highest and middle SBS values showed predominately a mixed-type failure mode, except the middle SBS value corresponding to the cervical third, which failed adhesively. The lowest bond strength values displayed an adhesive-type failure between the dentin

Table 1. Mean shear bond strength value by different location in the coronal dentin (CI-confidence interval, SE-standard error).

\begin{tabular}{ccc}
\hline Location in Dentin & Mean (SE) & $95 \%$ CI \\
\hline All & $38.8(1.5)$ & $35.7-41.9$ \\
Incisal & $36.9(3.1)$ & $30.7-43.1$ \\
Middle & $42.6(2.6)$ & $37.3-47.8$ \\
Cervical & $37.1(2.1)$ & $32.8-41.3$ \\
\hline
\end{tabular}

Table 2. Nonparametric Kruskal-Wallis rank test (Chi-square = 3.32 , degree of freedom $=2, p=0.19$ ).

\begin{tabular}{ccc}
\hline Location & Observation & Rank Sum \\
\hline Incisal & 20 & 589.0 \\
Middle & 20 & 719.5 \\
Cervical & 20 & 521.5 \\
\hline
\end{tabular}

Table 3. Mode of failure for representative SBS values for the incisal, middle and cervical crown thirds.

\begin{tabular}{cccc}
\hline Location & Value & SBS (MPa) & Mode of Failure \\
\hline Incisal & Highest & 51.70 & Mixed \\
Incisal & Middle & 35.90 & Mixed \\
Incisal & Lowest & 19.80 & Adhesive (DBA*/dentin) \\
Middle & Highest & 59.30 & Mixed \\
Middle & Middle & 39.50 & Mixed \\
Middle & Lowest & 17.30 & Adhesive (DBA $/$ dentin) \\
Cervical & Highest & 59.80 & Mixed \\
Cervical & Middle & 38.90 & Adhesive (DBA $*$ dentin) \\
Cervical & Lowest & 19.50 & Adhesive (DBA*/dentin) \\
\hline
\end{tabular}

* DBA = dentin bonding agent. 
and adhesive regardless of the crown location. Representative images for the described failure modes are shown in Figure 2.

Scanning electron microscope images of dentinal tubules cross-sectional view obtained from the different crown regions are depicted in Figure 3. The cross-sectional images show that dentinal tubules are oriented approximately at $90^{\circ}$ to the DEJ in superficial dentin irrespective of the crown third. Despite the relatively similar orientation, a somewhat rounder cross-section was observed for the middle third relative to the incisal and cervical thirds, which appeared rather oblique in cross-section.

\section{DISCUSSION}

This pilot study evaluated the shear bond strength of specimens bonded to different crown regions of superficial bovine incisor dentin. The main objective was to evaluate the influence of the variable "location of bonding" on bond strength. All other variables also known to affect the bond strength, namely surface preparation, adhesive strategy, test set up, storage conditions and several others, remained unchanged. Overall, high bond strengths were demonstrated irrespective of the crown third. Higher bond strengths were detected when bonding to the middle third (42.6 MPa) relative to the incisal (36.9 MPa) and cervical thirds (37.1 MPa). However, no significant differences between the crown thirds were evidenced with the sample size and power conditions used in this pilot study confirming the null hypothesis. Twenty specimens per group were used, which doubles the sample size of ten commonly used in bond strength studies $[14,20]$. However, no significant differences were observed between crown thirds suggesting that bonding to different crown regions yields no significant effect on the bond strength provided that the same dentin depth is used. In this regard, the use of bovine dentin represents an advantage due to their overall greater availability and

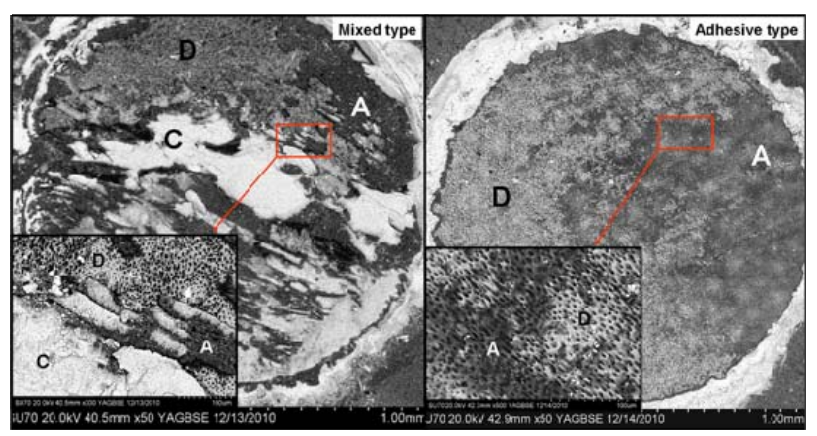

Figure 2. FE-SEM images in backscattered scanning electron mode at high and low magnification of "mixed-type" failure across the different interfacial layers and "adhesive" failure between dentin and adhesive $(\mathrm{C}=$ composite; $\mathrm{D}=\operatorname{dentin}$; $\mathrm{A}=$ adhesive).

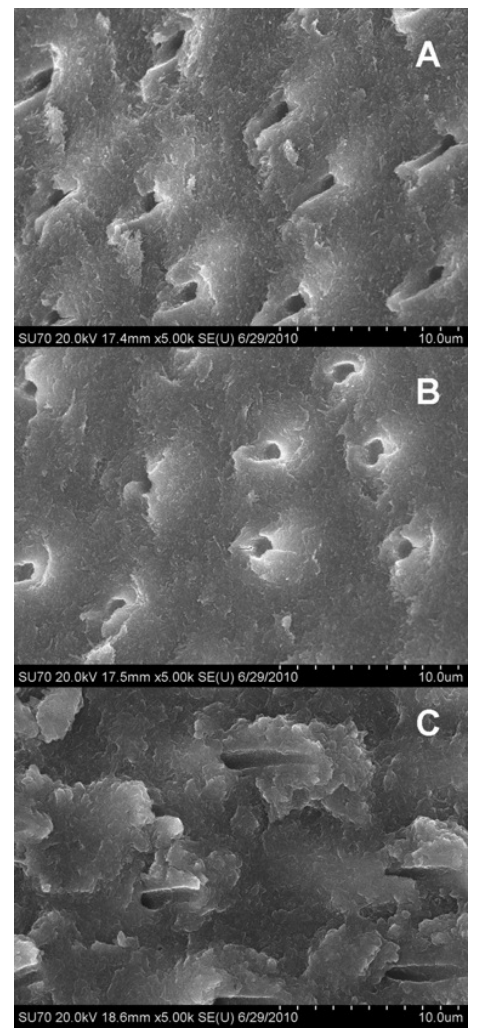

Figure 3. FE-SEM images in secondary electron mode display dentinal tubule orientation for the different crown thirds. (A) incisal third; (B) middle third; (C) cervical third.

considerably larger size, which allows a greater number of specimens to be obtained from each crown. Future studies should be undertaken using larger sample size and power, as these may be able to detect significant differences at the 0.05 level.

Our study was limited to the baseline SBS values, that is the loading of the specimens was done after 24 hours of bonding. It is likely that different results could have emerged from incorporation of the aging variable in the SBS evaluation; however given that considerably long storage periods are needed to detect the effects of hydrolytic degradation when using specimens of large surface area such as those used in shear bond strength tests [21], and considering the nature of the pilot study under investigation, baseline bond strength values were deemed to provide the desired information.

Similar to our results, previous studies have shown no significant effect of the tubule orientation on bond strength $[4,18,22]$. Phrukkannon evaluated micro-tensile strength using premolar teeth while Watanabe used third molars and Cehreli used primary teeth. Despite variations in the methodology and study design, the same notion was confirmed and no significant differences were detected when bonding to tubules oriented parallel, ob- 
lique or perpendicular to the surface. Conversely, other studies have shown that tensile strength of the mineralized dentin matrix is dependent on tubule orientation with the highest strength observed when the load is applied perpendicular to the direction of the tubules [23, 24]. A study by Lertchirakarn demonstrated that ultimate tensile strength was lowest when the tensile force was parallel to tubule orientation, and greatest at $90^{\circ}$ to tubule orientation [25]. This can be explained by the structural arrangement of dentin's collagen fibrils which are interwoven and arranged perpendicular to tubules [26], and apatite crystals which tend to be positioned parallel with the long axis of collagen fibrils. Thus, fractures perpendicular to the tubules occur predominantly within the plane of the collagen network, while fractures parallel to the tubules require disruption of the collagen fibrils.

Our descriptive observations of the cross-sectional view of the tubules at the incisal, middle and cervical thirds were intended to add to the bond strength results and provide a rationale to explain the observed results of no significant differences between crown thirds. FESEM images depicted in Figure $\mathbf{3}$ show that despite the zigzag course of the dentinal tubules from the pulp chamber to the DEJ, their cross-section, at least in superficial dentin, appears to be similar at the different crown thirds and oriented approximately at $90^{\circ}$ to the DEJ. This suggests that the different crown regions may exhibit similar mechanical properties [15]. A somewhat rounder cross-section was observed for the middle third relative to the incisal and cervical thirds, which appeared rather oblique in cross-section. Coincidently, higher bond strengths were demonstrated for the middle third relative to the incisal and cervical third perhaps indicating longer resin tag formation and consequently better strength.

\section{Observations on Failure Mode}

The present study started out as a basic morphological evaluation of the bond strength when bonding to different crown regions to determine the isotropic/anisotropic properties of bovine superficial dentin relative to shear bond strength. Our SEM observations soon made evident the greater complexity of the adhesive interface reported in previous studies $[3,27]$. The use of nominal values to report on the strength of adhesive interfaces has beenpreviously questioned due to the heterogeneity of the stress distribution and the number of variables known to play a role on the strength of the bonded interface [28, 29]. Hence, results from bond strength studies require cautious interpretation since they report on a number of variables relative to the adhesive and substrate, test set up and storage conditions rather than on the strength of the adhesive itself [20].

Analysis of the failure mode of mineralized tissues has gained attention more recently as it may provide a better understanding of these complex adhesive interfaces, their mechanical behavior, and the characteristic patters governing the fracture behavior [30]. Arecent review revealed that failure mode was reported in only $64 \%$ of the studies with $47 \%$ of these using stereomicroscope and only $17 \%$ using scanning electron microscope for observation of the failed interfaces [20]. FE-SEM in backscattered electron mode was used for our observations. In backscattered imaging mode, the contrast in the image is principally determined by average atomic number of the sample which allows identification of the different components present at the interface by observation of the contrast in grayscale images. As a result, tooth structure (containing calcium and phosphorus), adhesive (primarily organic) and resin composite (containing inorganic fillers of high atomic number as radioapicifiers) can readily be distinguished. In the system under investigation, the resin composite appears bright due to the zirconia filler particles present in Z100, the dentin appears of an intermediate gray level due to the calcium, phosphorus and oxygen content, and the adhesive appears dark since its composition is primarily unfilled organic material. Figure 2 depicts a mixed-type failure across the different interfacial layers. A central region of cohesive failure in the resin composite can be recognized, with bare dentin at the top and bottom area of the composite and adhesive resin also shown to the right side of the image. This specimen had an SBS value of $59.3 \mathrm{MPa}$, at the high end of the values obtained in this study. In agreement with our observations, other studies have demonstrated a positive correlation between bond strength values and the area of cohesive failure observed in mixed-type failures [31,32]. The development of improved dentin adhesive systems yield higher bond strengths, which have been shown to develop non-uniform stress distribution causing primarily cohesive failures on either substrate or composite precluding an accurate assessment of the true interfacial strength [31,32]. Figure 2 also illustrates an adhesive-type failure between the dentin and the adhesive. This specimen had a SBS value of $19.8 \mathrm{MPa}$, at the lower end of the values obtained in this study.

Although our observations of the failed interfaces were merely descriptive, certain trends were evidenced. Lower SBS values showed predominantly adhesive-type failures whereas higher SBS values showed primarily mixed failures through the body of the adhesive interface. These trends suggest that the entire adhesive assembly may behave stronger when acting as a single body rather than as separate layers. The intimacy of the interactions between dentin, adhesive and composite may be such that it surpasses the cohesive strength of each of its individual components and the adhesive strength between them. A number of studies have shown a strong positive 
correlation between bond strength values and the area of cohesive failure in resin observed in mixed failures [3, 32]. Our observations suggest that future studies are needed to gain a better understanding of the complex mechanism of adhesion and how these interfaces fail. It has been reported that a crack typically propagates from a critical size flaw found in an area subjected to high stresses [33]. However, the presence of mixed failures where there is a combination of cohesive and adhesive failures within the same bonded area, and how an adhesive bond transitions from a strong bond which exhibits cohesive failure to a weak bond which exhibits adhesive failure require further investigation. The load application at a single point on time, as in bond strength studies, is also not accurate representation of the repetitive stress conditions that teeth undergo during clinical function. The progressive and localized structural damage that takes place when the material is subjected to cyclic loading, such as during fatigue testing, would much better explain the failure mechanism of adhesive interfaces during clinical function [33]. Furthermore, a standard assessment method for evaluation of the failure of these interfaces, as well as a universal classification system of the different failure modes is needed for fair comparisons among studies to be feasible.

The anisotropic properties of dentin at different depths are generally accepted as confirmed by several studies. [19] However, no reference has been made to the anisotropic properties of dentin at one same depth in different crown regions. It may be speculated that variations in tubular orientation and number of tubules per surface area in different crown regions could affect the bond strength values, but this assumption has not been confirmed. The results of our study indicate that these aspects can be ignored. The absence of significant differences in shear bond strength values between the different crown thirds indicate that, regardless of tubule orientation, any crown region can be bonded when superficial bovine incisor dentin is used as substrate for shear bond strength testing. Clinically, this represents a major advantage considering the large size of bovine teeth, which allows fabrication of multiple specimens from a single crown.

\section{CONCLUSIONS}

Within the limitations of this study, the following conclusions may be drawn:

- The anisotropic behavior of superficial bovine dentin relative to bond strength was not confirmed. Dentinal tubular orientation in the different crown regions was similar and approximately at $90^{\circ}$ to the DEJ.

- Although no significant differences in SBS were detected, higher strengths to the middle third of superficial bovine coronal dentin were observed relative to the incisal and cervical thirds. This could be attributed to the sample size and power conditions used in the present pilot study. An increase in sample size may help detect significant differences in bond strength among the different crown thirds confirming the observed trends.

- Observation of the failure mode of representative specimens demonstrated that specimens with high bond strength values exhibited primarily mixed-type failures whereas low strength specimens exhibited adhesive failures between the dentin and adhesive. These trends indicate that evaluation of the fracture mechanics can provide much better insight on the functioning of these complex adhesive interfaces.

\section{ACKNOWLEDGMENTS}

The authors would like to thank Mr. Manthan Patel for his contribution with the statistical analysis and Mr. Peter Bush for his assistance with the FE-SEM processing and imaging procedures.

\section{REFERENCES}

[1] De Munck, J., Van Landuyt, K., Peumans, M., Poitevin, A., Lambrechts, P., Braem, M. and Van Meerbeek, B. (2005) A critical review of the durability of adhesion to tooth tissue: Methods and results. Journal of Dental Research, 84, 118-132.

doi:10.1177/154405910508400204

[2] Pashley, D.H. and Carvalho, R.M. (1997) Dentine permeability and dentine adhesion. Journal of Dentistry, 25 355-372. doi:10.1016/S0300-5712(96)00057-7

[3] Leloup, G., D’Hoore, W., Bouter, D., Degrange, M. and Vreven, J. (2001) Meta-analytical review of factors involved in dentin adherence. Journal of Dental Research, 80, 1605-1614. doi:10.1177/00220345010800070301

[4] Watanabe, L.G., Marshall, J.G.W. and Marshall, S.J. (1996) Dentin shear strength: Effects of tubule orientation and intratooth location. Dental Materials, 12, 109-115. doi:10.1016/S0109-5641(96)80077-7

[5] Marshall Jr., G.W., Marshall, S.J., Kinney, J.H. and Balooch, M. (1997) The dentin substrate: Structure and properties related to bonding. Journal of Dentistry, 25, 441-458. doi:10.1016/S0300-5712(96)00065-6

[6] Burke, F.J., Hussain, A., Nolan, L. and Fleming, G.J. (2008) Methods used in dentine bonding tests: An analysis of 102 investigations on bond strength. European Journal of Prosthodontics and Restorative Dentistry, 16, 158-165.

[7] Tagami, J., Tao, L., Pashley, D.H. and Horner, J.A. (1989) The permeability of dentine from bovine incisors in vitro. Archives of Oral Biology, 34, 773-777. doi:10.1016/0003-9969(89)90027-7

[8] Reinhard, S., Jörg, A.L., Oskar, B. and Werner, G. (2000) Comparison of the number and diameter of dentinal tubules in human and bovine dentine by scanning electron microscopic investigation. Archives of Oral Biology, 


\section{5, 355-361. doi:10.1016/S0003-9969(00)00006-6}

[9] Nakamichi, I., Iwaku, M. and Fusayama, T. (1983) Bovine teeth as possible substitutes in the adhesion test. Journal of Dental Research, 62, 1076-1081. doi:10.1177/00220345830620101501

[10] Reis, A.F., Giannini, M., Kavaguchi, A., Soares, C.J. and Line, S.R. (2004) Comparison of microtensile bond strength to enamel and dentin of human, bovine, and porcine teeth. Journal of Adhesive Dentistry, 6, 117-121.

[11] Schilke, R., Lisson, J.A., Bauss, O. and Geurtsen, W. (2000) Comparison of the number and diameter of dentinal tubules in human and bovine dentine by scanning electron microscopic investigation. Archives of Oral Biology, 45, 355-361. doi:10.1016/S0003-9969(00)00006-6

[12] Saunders, W.P. (1988) The shear impact retentive strengths of four dentine bonding agents to human and bovine dentine. Journal of Dentistry, 16, 233-238. doi:10.1016/0300-5712(88)90080-2

[13] Reeves, G.W., Fitchie, J.G., Hembree Jr., J.H. and Puckett, A. D. (1995) Microleakage of new dentin bonding systems using human and bovine teeth. Operative Dentistry, 20, 230-235.

[14] Inoue, T., Takahashi, H. and Nishimura, F. (2002) Anisotropy of tensile strengths of bovine dentin regarding dentinal tubule orientation and location. Dental Materials Journal, 21, 32-43. doi:10.4012/dmj.21.32

[15] Kinney, J.H., Marshall, S.J. and Marshall, G.W. (2003) The mechanical properties of human dentin: A critical review and re-evaluation of the dental literature. Critical Reviews in Oral Biology and Medicine, 14, 13-29.

[16] Pashley, D., Okabe, A. and Parham, P. (1985) The relationship between dentin microhardness and tubule density. Endodontics and Dental Traumatology, 1, 176-179. doi:10.1111/j.1600-9657.1985.tb00653.x

[17] Schüpbach, P., Krejci, I. and Lutz, F. (1997) Dentin bonding: Effect of tubule orientation on hybrid-layer formation. European Journal of Oral Sciences, 105, 344352. doi:10.1111/j.1600-0722.1997.tb00251.x

[18] Phrukkanon, S., Burrow, M.F. and Tyas, M.J. (1999) The effect of dentine location and tubule orientation on the bond strengths between resin and dentine. Journal of Dentistry, 27, 265-274. doi:10.1016/S0300-5712(98)00060-8

[19] Smith, D.C. and Cooper, W.E. (1971) The determination of shear strength. A method using a micro-punch apparatus. British Dental Journal, 130, 333-337. doi:10.1038/sj.bdj.4802665

[20] Braga, R.R., Meira, J.B., Boaro, L.C. and Xavier, T.A. (2010) Adhesion to tooth structure: A critical review of “macro" test methods. Dental Materials, 26, e38-e49. doi:10.1016/j.dental.2009.11.150

[21] Kiyomura, M. (1987) Bonding strength to bovine dentin with 4-META/MMA-TBB resin long-term stability and influence of water. Journal of the Japanese Society for Dental Materials and Devices, 6, 860-872.

[22] Cehreli, Z.C. and Akca, T. (2003) Effect of dentinal tubule orientation on the microtensile bond strength to primary dentin. Journal of Dentistry for Children, 70, 139-144.

[23] Carvalho, R.M., Fernandes, C.A., Villanueva, R., Wang, L. and Pashley, D.H. (2001) Tensile strength of human dentin as a function of tubule orientation and density. Journal of Adhesive Dentistry, 3, 309-314.

[24] Inoue, S., Pereira, P.N., Kawamoto, C., Nakajima, M., Koshiro, K., Tagami, J., Carvalho, R.M., Pashley, D.H. and Sano, H. (2003) Effect of depth and tubule direction on ultimate tensile strength of human coronal dentin. Dental Materials Journal, 22, 39-47. doi:10.4012/dmj.22.39

[25] Lertchirakarn, V., Palamara, J.E. and Messer, H.H. (2001) Anisotropy of tensile strength of root dentin. Journal of Dental Research, 80, 453-456. doi:10.1177/00220345010800021001

[26] Sogaard-Pedersen, B., Boye, H. and Matthiessen, M.E. (1990) Scanning electron microscope observations on collagen fibers in human dentin and pulp. Scandinavian Journal of Dental Research, 98, 89-95.

[27] Nakabayashi, N., Nakamura, M. and Yasuda, N. (1991) Hybrid layer as a dentin-bonding mechanism. Journal of Esthetic and Restorative Dentistry, 3, 133-138. doi:10.1111/j.1708-8240.1991.tb00985.x

[28] Van Noort, R., Noroozi, S., Howard, I.C. and Cardew, G. (1989) A critique of bond strength measurements. Journal of Dentistry, 17, 61-67. doi:10.1016/0300-5712(89)90131-0

[29] DeHoff, P.H., Anusavice, K.J. and Wang, Z. (1995) Three-dimensional finite element analysis of the shear bond test. Dental Materials, 11, 126-131. doi:10.1016/0109-5641(95)80047-6

[30] Yan, J., Clifton, K.B., Reep, R.L. and Mecholsky Jr., J.J. (2006) Application of fracture mechanics to failure in manatee rib bone. Journal of Biomechanical Engineering, 128, 281-289. doi:10.1115/1.2187044

[31] Pashley, D.H., Sano, H., Ciucchi, B., Yoshiyama, M. and Carvalho, R.M. (1995) Adhesion testing of dentin bonding agents: A review. Dental Materials, 11, 117-125. doi:10.1016/0109-5641(95)80046-8

[32] Al-Assaf, K., Chakmakchi, M., Palaghias, G., KaranikaKouma, A. and Eliades, G. (2007) Interfacial characteristics of adhesive luting resins and composites with dentine. Dental Materials, 23, 829-839. doi:10.1016/j.dental.2006.06.023

[33] Loughran, G.M., Versluis, A. and Douglas, W.H. (2005) Evaluation of sub-critical fatigue crack propagation in a restorative composite. Dental Materials, 21, 252-261. doi:10.1016/j.dental.2004.04.005 